\title{
Analysis of Logical Path of Construction of Sports Postgraduates' Academic Integrity System
}

\author{
Guibin $\mathrm{Su}$ \\ Sports Science College \\ Mudanjiang Normal University \\ Mudanjiang China
}

\author{
Cheng Cao \\ Physical Education Department \\ Mudanjiang Medical University \\ Mudanjiang China
}

\begin{abstract}
Academic integrity system of sports postgraduates proceeds from the public's demand whose formation is a dynamic and changing process. It is the result of continuous selection, running-in, identification and inspection between postgraduate education and the public. From economics analysis of the generation and running of academic integrity system of sports postgraduates, this paper emphasizes that the production process of academic integrity system of sports postgraduates is the result of games. On this basis, the author puts forward the ways of eliminating sports postgraduates' academic integrity crisis and building Chinese academic integrity system of sports postgraduates, that is to make efforts to create good external environment and system restriction for the multiple games between postgraduates education and the public and at the same time to increase the cultivation of integrity of our postgraduates education main body namely postgraduates cultivation units and postgraduates themselves and to continuously establish the academic integrity system of sports postgraduates which adapts to market economy and is perfect.
\end{abstract}

Keywords-postgraduate education; multiply games; integrity system; economics analysis; path; system restriction

Sports postgraduate education in China has the tendency of cooperation and in harmony with the public since its birth. The development of integrity relationship between postgraduate education and the public is always built on the basis of common understanding and value orientation. How to set up a complete set of sports postgraduate's academic integrity mechanism in an environment of mutual trust and honest between the public and cultivation units of postgraduates is a problem that theory and reality must face. From the perspective of embryology, the academic integrity system of sports postgraduates proceeds from the public's demand whose formation is a dynamic and changing process. It is the result of continuous selection, running-in, identification and inspection between postgraduate education and the public.

\section{THE FOUNDATION OF CONSTRUCTION OF OUR ACADEMIC INTEGRITY SYSTEM OF SPORTS POSTGRADUATE EDUCATION}

\section{A. Common Benefits of Postgraduates and Cultivation Units of Postgraduates}

For cultivation units of postgraduates, the behavior of the public purchasing products offered from it can be treated as a deal between the public and the units. Benefits are the basic motive of this deal which is done on the basis of the same or similar benefits between the public and cultivation units of postgraduates. The more consistent the benefits of both sides are, the easier the deal can be reached, that is the deal basis is common benefits. Because, only in this way can both sides have chances and motive power to trust each other and believe that the other side will bring long term and maximum benefits. Maybe at first the benefits are not clear but there are enough reasons to let the public and cultivation units of postgraduates make the choice: I trust you because I can see the prospective benefits. Same or similar benefits contribute to the possibilities of formation of the deal and occurrence of mutual integrity relationship. The formation of intangible benefits and enhanced mutual integrity relationship between the public and cultivation units of postgraduates further promote the consistent degree of intangible benefits of each other.

\section{B. Dynamics and Complementarities of Academic Integrity Relationship Between Postgraduates and Cultivation Units of Postgraduates}

The development of integrity relationship between the public and cultivation units of postgraduates is dynamic which needs the latter to influence the public with large and real integrity events. The directions of this influence can be direct or indirect. The process of integrity happening is generally along the logical path of suggesting integrity signal, showing integrity tendency, questioning the integrity, confirming and following integrity. At the early stage of postgraduate education development, cultivation units of postgraduates are at the initial stage of suggesting, showing integrity signals and tendency, which inevitably causes some public' doubts. Thus, the public needs a period of waiting and seeing to make sure the motive and real purpose of cultivation units of postgraduates. Therefore, units require lots of integrity events and action to make the public on longer doubt and truly accept the sincerity or the basis of trust is not solid. And ongoing academic integrity events of cultivation units of postgraduates will filter the public's suspicions and make the integrity relationship move to next stage. At the same time, the influence strength should be large enough, which directly decides the strength of the public's trust and trust psychology. Weakening of influence strength of cultivation units will destroy the trust psychology tendency of the public on cultivation units which is formed before and affect the integrity happening process of cultivation units. 
In addition, the deal between the public and cultivation units of postgraduates is the touchstones of testing the integrity of both sides. Both need to constantly keep a promise to trade for each other's trust. That is to say, the maintenance of integrity is mutual and either side giving up or negative could lead to interrupt of this continuously developed integrity relationship which will make the deal between both sides lose rational basis. This will cause integrity relationship between both sides to loss its reason to exist.

\section{ECONOMIC ANALYSIS OF THE BUILDING OF ACADEMIC INTEGRITY RELATIONSHIP BETWEEN POSTGRADUATES AND CULTIVATION UNITS OF POSTGRADUATES}

According to the above analysis, from the perspective of economics, the formation process of integrity relationship between the public and units is a game behavior of both sides based on their own benefits. "In the case of little game playing, going back on one's word will become the Nash equilibrium of both sides and in the case of much game playing, keeping one's word will become the Nash equilibrium of game." In the process of matrix analysis of game benefits, for the public, there are two choices: trusting or distrusting cultivation units of postgraduates. Cultivation units of postgraduates also have choices of breaking or keeping their word. If the public choose to distrust cultivation units then the game profit is zero where Nash equilibrium will not create integrity relationship. Because there is no deal happened between two sides. However, if the public choose to trust strategy of cultivation units then the game between two sides moves to the second stage. At this stage, it is the cultivation units to make a choice: choosing to break their word will get two units of profit and the public's profit is zero; choosing to keep their word will get one unit of profit and the public get one. Due to asymmetric information of both sides and based on the consideration of maximum profit, cultivation units based on "rational economic man" would choose to go back one their word and gain two units of profit. But the consequence of this choice is that the deal between both sides has to been stopped because of cultivation units' going back on their word. If before the deal the public can predict this, they will choose to distrust the cultivation units. The reason is that the public not only cannot get one unit of profit, their will lose one. Also, cultivation units won't obtain one unit of profit. If the circumstances are always so, the cooperation between the two sides don't take effect, and the Nash equilibrium is zero and so the benefits obtaining path stops.

The tendency of "rational economic man" of cultivation units of postgraduates leads to the deal between the public and units a dead end. The capital flow of the entire higher education system will decrease and the overall benefits of higher education system appear as "irrational" equilibrium that is Nash equilibrium is zero profit instead of Pareto optimal state. Based on the above analysis we can see that seeking the rational overall optimization of the public and cultivation units of postgraduates, that is each side gets one unit of profit which inevitably require both sides to achieve logical conjunction of trust and trustworthiness instead of the trend of distrust and breaking faith. This preset support of integrity relationship of the public and cultivation units will inevitably bring long-term cooperation profits namely one unit of profit for each side. Therefore, the formation and strengthening of integrity relationship need both sides deducing by game. The deeper the game, the more firmly the integrity cooperation foundation is. Any a discreditable behavior between both sides will lead to situation of zero profit. At this time, credibility crisis occurs and lays foundation for the next unsuccessful game for both sides.

\section{THE REASONABLE PATH OF BUILDING ACADEMIC INTEGRITY SYSTEM OF OUT SPORTS POSTGRADUATES}

\section{A. Establish Modern Culture of Academic Integrity}

First, higher education management departments should take the lead in demonstration and practice for commitment of "openness, fairness and justice" consistent with the spirit of education. Those inaction, negative action and illegal action should be investigated for administrative and legal responsibilities. Higher education management departments should cultivate positive administrative ethics culture and bring its integrity level into their performance management system. All levels of higher education institutions should establish integrity assessment criteria and improve the self-discipline consciousness and concept, the higher education administrative management departments should constantly enhance their credibility and affinity. At the same time, they should constantly change their function turning to administrative ideas of "service" and "response" and run integrity quality as "intangible assets". On operation level of concrete system, the main task of higher education management departments is to build a fair and impartial higher education honesty culture environment in the purpose of making higher education information transparent and solving the problem of poor integrity environment for cultivation units of postgraduates caused by information asymmetry. On the training target, their core is to cultivate awareness of integrity and dedication of the staff of higher education management departments and foster independent administrative ethics personality and strengthen administrative responsibility consciousness. On the level of system, higher education management departments should establish personal integrity archives and audit system of the key and sensitive positions. Those administrative behaviors contrary to higher education integrity philosophy and irresponsible for the public and cultivation units of postgraduates should be dealt with seriously. Higher education management departments should gradually establish modern administrative ethics consciousness and improve the spiritual implication of credit quality in practice and temper their integrity in specific administrative act.

Second, credit system construction of cultivation units of postgraduates should be perfected and "Mark System of Cultivation Units of Postgraduates" should be fully implemented. We should try to make the credit information of cultivation units open and transparent and constrain credit behaviors of cultivation units by market force. Credit mark of cultivation units is a kind of "brand effect" and "intangible 
assets" and also the embodiment of integrity culture in cultivation units. To some extent, credit mark is the "lifeline" of cultivation units and has the function of supervision and correction for planning and restricting behaviors of cultivation units.Implementation of "Mark System of Cultivation Units of Postgraduates" is beneficial to urge cultivation units to fulfill a contract in accordance with the law and agreement. The concrete construction of such system should be guided by the higher education management departments and cooperated by cultivation units of postgraduates and others. In terms of financial management, auditing management, market behavior, cultivation units of postgraduates should create demand for credit information from the system and exemplarily lead recognition of "Mark System of Cultivation Units of Postgraduates" for higher education system. At the same time, we should learn and draw lessons from treatment of lack of honesty of foreign cultivation units of postgraduates, such as establishing "Specifications of Cultivation Units of Postgraduates" among cultivation units to deepen degree of trust between each other and cultivation units with civil society in order to continuously broaden and strengthen occurrence of space of integrity system for cultivation units and to breed higher education credit system. Moreover, in terms of integrity quality cultivation of the related officials, we should base on the training of complete integrity personality and strengthen cultivation path of education and internalization path from babies. Postgraduates or their cultivation units who have done good practice in integrity rules should be awarded in order to play a positive exemplary role by being set up as honest models.

\section{B. Improving Complete Rigid Laws and Regulations of System of Postgraduate Academic Integrity}

"A general just implementation of rule of law provides basis of mutual cooperation and disputes solving for unrelated strangers, which can expand the radius of trust" on a large scale. The real fair implementation of rule of law needs complete legal system to ensure that the game between two sides is done smoothly. The legal system here not only refers to the concrete and basic legal system and provisions but also includes relevant credit legislation. Specifically, legislation of integrity ontology should be contained such as law of protection of integrity etc and procedure act of integrity, such as remedy for integrity and punishment methods. Among these laws the power and obligation of cultivation units of postgraduates should be constantly improved to make clear of legal relationship of the deal between cultivation units and the public. We should overcome the deal state of unclear property rights and responsibility of some cultivation units of postgraduate to impel standardization, institutionalization and legalization of the deal between two sides. At the same time, increasing the legislation of integrity information and making legal and rational interpretation for collection, disclosure, feedback, repairment of integrity information of cultivation units of postgraduates to help to avoid obsession of asymmetric information for both sides and ensure that cultivation units survive and develop in the competitive environment of fair and open. At the same time, relevant law and policy authorities needs keeping pace with the times to reintegrate higher education law, anti-unfair competition law and a series of related laws. We should make full use of existing, advanced legal system to reform legal concepts that are not suitable for market economy and make rational interpretation which is pervasive for the building of academic integrity system of cultivation units of postgraduates. The legal environment of asserting integrity for cultivation units and postgraduates should be constantly set up and improved to form a virtuous operations system of higher education in which everyone is honest.

\section{Setting Up Academic Integrity Management Institutions for Sports Postgraduates}

Practice of countries whose higher education systems have developed more completely proves that single higher education integrity management institutions can indecently excise it function and has the advantages that administrative functions do not have. Independent higher education integrity management institutions should include system pattern, form of organization, management system, which can take advantage of modern advanced techniques to build network platform for higher education integrity system to establish socialized credit archives of cultivation units of postgraduate. Such credit archives are mainly composed of honest credit, illegal record and industry record of cultivation units and postgraduates. Such credit archives make everyone within the higher education system have complete integrity record which record personal events and experiences of breaking or keeping faith. Such credit archives not only make the "breaking" have damage in academic achievement and economic interests, but also make those who dare to defy the law pay expected cost of losing credit. On this basis, the constantly amended credit record of cultivation units of postgraduates and postgraduates themselves make everyone within higher education system dare not deviate.

On organization and management of higher education credit problem, industry self-discipline should be the first to be done. Warming education for higher education integrity problem and establishing mutual discipline organization of within the industry of higher education, evaluating the management institutions of higher education, building different information management platform help to each public learning about and refer to the circumstances of keeping faith of cultivation units of postgraduates and individuals through network and the media to gradually form a situation in which there are industry self-discipline, mutual discipline and the public involved in common supervision.On authority of credit management of cultivation units of postgraduates, first, the authority should be separated from the administrative institutions of higher education, giving full play to the advantages of independent credit management mechanism to preventing the infiltration of administrative power and human intervention. Constantly improving the performance evaluation system of higher education integrity, monitoring and detecting credit risk expectations of individuals and cultivation units and 
predicting development trend of credit characteristics provide dynamic information resources and effective methods for credit management of higher education.

Finally, credit management institutions of higher education should bear the function of public release and feedback of relevant information, which requires institutions to publish integrity record of cultivations unit of postgraduates, postgraduates and relevant officials through various media at regular intervals to control the occurrence of related moral hazard and adverse selection.

\section{Increasing the Strength of Punishing and Supervising of Discreditable Cultivation Units of Sports Postgraduates and Individuals}

For cultivation units, the less the cost of dishonest behaviors is than relevant profit, the bigger probability of occurrence is for cultivation units. Therefore, increasing the strength of penalties for the dishonest behaviors of cultivation units is the direct means and also the main content of compensation mechanism for most trustworthy cultivation units of postgraduates. For dishonest cultivation units, the penalties can be direct material punishment or forcing them to apologize through news media and make corresponding guarantee for future behaviors. In this foreign practice gives us a good enlightenment. Taking Italy, South Korea's for example, academic fraud after the investigation and confirmation of judicial organ, the higher education management departments first impose hefty penalties and a series of fines on the dishonest and fraud and then ask the person in charge to apologize on specified media and guarantee that there is no repeat. The operation process of market economy is the chain for frequently occurrence of credit. Once a chain stopped, it is difficult for each part of the whole system to play a normal role. Therefore, every key link of cultivation units must be strictly supervised. Taking advantage of modern media technology and network techniques to put the postgraduate operating behaviors under high dense regulation can effectively forcing cultivation units to follow a fair, fair trading rules and procedures. For the doubtable parts should be given a timely warming to prevent the occurrence of dishonest behaviors.

\section{CONCLUSION}

The building of academic integrity system of sports postgraduates in China is a process of constant development, which needs constantly summing up experience and absorbing foreign advanced concept of integrity and successful integrity management technology to establish higher education credit standards and value mode suitable for Chinese national conditions and improving integrity management that adapts to the overall development of market economy and is accepted by higher education integrity culture form. While creating honest external environment for cultivation units of postgraduates, management institutions of higher education should focus on the cultivation of integrity consciousness of cultivation units, from two aspects of value rationality and instrumental rationality, comprehensively shaping the atmosphere of credit behaviors and the concepts to gradually perfect credit value and means of higher education system.

\section{REFERENCES}

[1]. [USA] Robert D. Putnam. Making Democracy Work: Civic Traditions in Modern Italy $[\mathrm{M}]$. translated by Wang Lie and Lai Hairong. Nangchang: Jiangxi people's publishing house, 2001: 200

[2]. Guo Jinguang, Gao Jingmei. Credit Deficiency and New Treating Methods $[\mathrm{J}]$. Journal of Chongqing University of Industry and Commerce, 2003(3).

[3]. Francis fukuyama. translated by Liu Bangli etc. Division: Reconstruction of human nature and social order [M]. Beijing: China Social Sciences Press, 2002:24, 305.

[4]. Anti-cheating Focusing on Management. GuangMing Daily, 19941205.

[5]. Start the Social Credit system in China, Beijing Youth Daily A23 edition, 2003116 